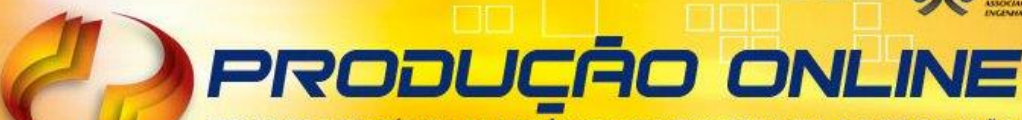 \\ REVISTA CIENTIFICA ELETRÔNICA DE ENGENHARIA DE PRODUÇÃO \\ ISSN 1676-1901
}

\section{ANALISANDO A RELAÇÃO ENTRE JUSTIÇA PERCEBIDA E SATISFAÇÃO DECLARADA PELOS CLIENTES APÓS FALHAS DE SERVIÇO: ESTUDO DE CASO EM UMA EMPRESA DE TELECOMUNICAÇÕES}

\section{ANALYZING THE RELATIONSHIP BETWEEN PERCEIVED JUSTICE AND SATISFACTION AFTER SERVICE FAILURES: A CASE STUDY IN A TELECOM COMPANY}

\author{
Marcus Augusto Vasconcelos Araúijo* E-mail: marcusaugusto77@hotmail.com \\ Marcos André Mendes Primo** E-mail: marcos.primo@faculdadeguararapes.edu.br \\ Jamille Queiroz Leite*** jamillequeizor@gmail.com \\ ${ }^{*}$ Faculdade Boa Viagem (FBV/MPGE) \\ ${ }^{* *}$ Faculdade Guararapes; Universidade Federal de Pernambuco (FG; UFPE/PROPAD) \\ ${ }^{* * *}$ Universidade Federal de Pernambuco (UFPE/PROPAD)
}

\begin{abstract}
Resumo: O defeito zero é algo praticamente inalcançável em operações de serviços, o que torna a capacidade das empresas de gerenciar eficazmente as reclamações dos clientes oriundas das falhas de serviços uma condição sine-qua-non para o seu sucesso no longo prazo. Este artigo tem como objetivo central avaliar a influência das percepções de justiça na satisfação dos clientes após a condução de um processo de gerenciamento de reclamações. Para tanto, foi analisado o caso de uma empresa de telefonia celular a partir da condução de uma survey com 496 clientes que reclamaram no seu call center. Foram avaliadas as percepções individuais de justiça declaradas pelos clientes e a sua relação com a satisfação após o processo. Os resultados obtidos por meio da aplicação de Regressão Logística demonstram que as percepções de justiça processual e distributiva influenciaram de forma significativa a satisfação, enquanto que a justiça interpessoal, apesar de ter obtido a melhor avaliação individual, não demonstrou ter relação significativa com este construto. São feitas reflexões relevantes baseadas nestes resultados, considerando que diversos elementos de justiça mal avaliados individualmente apresentaram grande influência na satisfação final e indicando a necessidade da empresa em rever suas prioridades em termos de revisão de processos e treinamento de funcionários.
\end{abstract}

Palavras-chave: Falhas de serviço. Teoria da Justiça. Satisfação.

Abstract: The zero defect is something virtually unachievable in service operations, which makes the ability of companies to effectively manage customer complaints derived from service failures an important condition for its success in the long term. This article evaluates the influence of justice perceptions in customer satisfaction after a complaints management process. The case of a cell phone company is analyzed in which a survey with 496 customers who complained to the firm's call center was applied. Customers' justice perceptions and its relationship with satisfaction after the procedure were evaluated. The results obtained with a logistic regression analysis indicated that perceptions of procedural and distributive justice influenced significantly satisfaction, while the interpersonal justice, despite having had the high individual mean, didn't showed any significant relationship with this construct. Some important reflections are made based on these results, considering that many elements of justice poorly evaluated had great influence on the final satisfaction, which indicated the need for the company to review its priorities in terms of processes evaluation and employee training.

Keywords: Service failures. Justice Theory. Satisfaction.

Revista Produção Online, Florianópolis, SC, v.16, n. 1, p. 126-150, jan./mar. 2016. 


\section{INTRODUÇÃO}

Uma característica específica da prestação de serviços é a simultaneidade da sua produção e consumo, tornando o resultado satisfatório da sua entrega dependente de vários fatores como a atitude e o preparo dos funcionários, a capacidade produtiva do sistema, o comportamento e a expectativa dos clientes, dentre outros. Neste contexto, as falhas parecem ser inevitáveis (SLACK et al., 1996), pois há uma grande quantidade de fatores que influenciam o processo e, alguns deles, não são controlados pelas empresas. Este argumento é fundamentado pelo fato de que "os erros são características inevitáveis de todo o esforço humano, incluindo a entrega de serviços" (BOSHOFF, 1997, p. 110).

Em situação de falhas, vários autores argumentam que deve ser operacionalizada uma recuperação de serviços de modo a reduzir os impactos negativos gerados pelo erro, tornando, assim, os consumidores tão ou mais satisfeitos do que a situação anterior ao ocorrido (ver, por exemplo, HART et al., 1990; SWANSON e KELLEY, 2001). Dessa forma, a recuperação de falhas se torna uma oportunidade para a empresa demonstrar qualidade mesmo em um momento de crise na sua relação com o cliente (ALMEIDA e TOLEDO, 2003).

Uma das teorias que melhor descreve as percepções dos clientes nas situações em que há conflitos destes com a empresa prestadora de serviços é a teoria da justiça (TAX et al. 1998), pois, no momento da reclamação, há a oportunidade da empresa re-equilibrar a justiça percebida na transação que foi afetada por uma falha (SMITH et al. 1999). Assim, após o processo de recuperação de serviços, quanto melhor a percepção de justiça por parte dos clientes, maior tende a ser o nível de satisfação observado (ver, por exemplo, TAX et al., 1998; SANTOS e FERNANDES, 2008).

Observa-se que boa parte do esforço de recuperação de falhas nas grandes empresas de serviço brasileiras, tem sido feito em operações de call centers, pois é neste setor que grande parte dos clientes faz as suas queixas, caracterizando estas operações como o principal ponto de contato das empresas com os seus clientes. Objetivando melhorar a qualidade das suas operações, os call centers conduzem sistematicamente treinamentos de seu pessoal e revisões de processos e 
procedimentos, utilizando como referencial básico os resultados das pesquisas de satisfação de clientes, conduzidas periodicamente pelas empresas prestadoras de serviços.

O objetivo das pesquisas de satisfação de clientes é mensurar o desempenho das empresas sob a ótica dos clientes, evidenciando as suas forças e fraquezas e permitindo, assim, que as oportunidades de melhorias identificadas sejam trabalhadas e a sua competitividade incrementada (ROSSI e SLONGO, 1998). Estas pesquisas, contudo, tendem a ter um caráter descritivo e normalmente analisam os itens pesquisados apenas em formatos univariados, ou seja, apenas a partir da apresentação das estatísticas descritivas de cada questão. Dessa forma, relações importantes entre construtos deixam de ser avaliadas, o que reduz as possibilidades de aplicação dos resultados encontrados e aumenta o risco associado à tomada de decisão, que pode não surtir o efeito desejado na melhoria da satisfação dos clientes.

Neste trabalho é apresentado um estudo conduzido no call center em uma empresa de telefonia celular que objetivou avaliar a relação entre justiça percebida e satisfação dos clientes após um processo de gerenciamento de reclamações. Neste sentido, é apresentada uma comparação das relações encontradas entre percepções de justiça e satisfação e com as avaliações individuais de cada dimensão de justiça percebida. Dessa forma, pretende-se evidenciar quais são os antecedentes de maior influência na satisfação e até que ponto os itens de melhores avaliações individuais realmente são os mais importantes para o resultado final do processo.

\section{FALHAS NA PRESTAÇÃO DE SERVIÇOS}

Apesar da constante busca pela qualidade na prestação dos serviços e todo esforço das empresas para que não ocorram falhas, as características únicas relacionadas à natureza dos serviços, como a sua heterogeneidade, a perecibilidade, a simultaneidade entre produção e consumo, tornam as falhas inevitáveis (LOVELOCK; WIRTZ; HEMZO, 2011), o que torna a habilidade das empresas em contornar a situação frente aos clientes um ponto vital na sua gestão organizacional (HART et al., 1990).

Revista Produção Online, Florianópolis, SC, v.16, n. 1, p. 126-150, jan./mar. 2016. 
As falhas em serviços consistem na "...percepção dos clientes de que um ou mais aspectos específicos da entrega do serviço não atenderam às suas expectativas." (LOVELOCK e WRIGHT, 2002, p. 161) e podem ser consequências, por exemplo, de ações dos funcionários, do fracasso em responder a necessidades específicas de clientes, do não cumprimento de promessas por parte da empresa, do serviço indisponível ou irracionalmente lento (BITNER et al., 1990). Kelly et al. (1993) reforçam a existência da multiplicidade de causas indicando que as falhas de serviço podem variar em intensidade e freqüência e podem resultar em clientes mais ou menos insatisfeitos.

Diversos estudos apontam que, quão mais críticas ou desagradáveis para o cliente são as falhas, menores são os níveis de satisfação observados (ver, por exemplo, BITNER et al., 1990, 1994; FREITAS, 2014; GILLY e GELB, 1982; GOODWIN e ROSS, 1992; HOFFMAN et al., 1995; HSU e CHIH, 2014; KOMUNDA e OSARENKHOE, 2012; MICHEL, 2001; OZGEN e KURT, 2012; SMITH et al., 1999; TSAI e WANG, 2014; WAQAS, ALI e KHAN, 2014; WEBSTER e SUDARAM, 1998; YANG e CHENG, 2014; ZHU et al., 2004), aumentando a tendência de sua saída da empresa.

Adicionalmente, verifica-se que após a ocorrência da falha, uma das opções do cliente é reclamar junto à empresa prestadora, o que permite que a empresa gerencie a reclamação, investigando as causas da falha, e operacionalize uma estratégia de recuperação de serviços, no intuito conduzi-lo novamente a um estado de satisfação (TAX et al., 1998).

Dessa forma, o modo pelo qual as reclamações são gerenciadas, exerce um impacto direto sobre a confiança que os clientes depositam no fornecedor, na satisfação percebida do serviço e, por conseguinte, no seu relacionamento com a empresa (SANTOS e FERNANDES, 2008), proporcionando uma oportunidade para a empresa alcançar a fidelização dos clientes que poderão atingir maiores níveis de satisfação do que caso o fracasso nunca tivesse ocorrido (MAGNINI, 2007). Opinião semelhante é apresentada por Silva e Lopez (2014), que ressaltam a importância do prestador de serviços perceber as reclamações como sendo uma oportunidade de solidificar e fortalecer o relacionamento com seus clientes.

Verifica-se que algumas das prescrições mais comuns em situações de falhas se concentram nas empresas se desculparem, repetirem o serviço e até oferecerem Revista Produção Online, Florianópolis, SC, v.16, n. 1, p. 126-150, jan./mar. 2016. 
uma compensação para os clientes, tentando, dessa forma, gerar uma solução paliativa em resposta ao problema gerado (LOVELOCK; WIRTZ; HEMZO, 2011). Battaglia e Borchardt (2010) verificaram que em empresas com alto grau de contato com o cliente, as ações de recuperação desenvolvidas são mais flexíveis e adaptáveis para situações específicas. Isso ocorre, sobretudo, nos casos em que a estrutura organizacional é reduzida, pois, dessa forma, o recebimento das queixas e insatisfações é facilitado, o que torna as ações de recuperação mais rápidas. Já em organizações com moderado grau de contato com o cliente final, as ações de recuperações tendem a serem menos ágeis, pois são dependentes dos programas de treinamento e devem seguir aos procedimentos internos da empresa. Por fim, nas organizações de baixo contato com o cliente, as ações de recuperação tem como prioridade obedecer ao estabelecido em normas e procedimentos regulamentadores, tornando-as, muitas vezes, não tempestivas, dada a sua baixa velocidade de operacionalização.

\subsection{As percepções de justiça no gerenciamento de reclamações}

Diversos estudos verificaram a importância do uso dos conceitos relacionados à teoria da justiça para a avaliação do gerenciamento de reclamações (ver, por exemplo, GILLILAND, 1993; GOODWIN e ROSS, 1990 e 1992; SANTOS e FERNANDES, 2008; TAX et al., 1998). Nesta linha, estudos relacionados à percepção de justiça em relações sociais, por exemplo, tem se concentrado na teoria da equidade, que considera a interação social composta por uma troca entre duas partes em que cada uma compara os insumos ("inputs") destinados à interação com os resultados ("outputs") obtidos. Assim, quando há um resultado favorável para uma das partes, esta tende a considerar a transação como justa enquanto que, se há um resultado desfavorável, a troca é entendida como injusta (SANTOS e FERNANDES, 2008).

Oliver e Swan (1989), em um estudo seminal, analisaram a influência das percepções de justiça na satisfação e desta na intenção de recompra no contexto de uma transação de vendas. O estudo enfocou a transação entre vendedor e cliente sob a ótica da equidade, verificando que há influência significativa da percepção de 
justiça distributiva (equidade) na satisfação com o processo de vendas e desta na intenção de recompra futura.

Goodwin e Ross (1990), por sua vez, ampliaram a aplicação do conceito de justiça analisando, além da justiça distributiva, os fatores relacionados à justiça interpessoal e processual dentro do contexto de gerenciamento reclamações. Os autores verificaram que, após a ocorrência de uma falha de serviço e a respectiva reclamação por parte do cliente, este avalia esforços, procedimentos e resultados advindos sob a ótica das três dimensões de justiça de forma simultânea. Este tipo de abordagem vem sendo utilizada em diversos estudos recentes, que tem confirmado o efeito das percepções de justiça na satisfação e na confiança após o gerenciamento de reclamações (ver, por exemplo, ASSEFA, 2014; BRATA e JULIANA, 2014; DEWITT, 2008; LIN, WANG e CHANG, 2011, OZGEN e KURT, 2012).

Ao se avaliar individualmente cada uma das três dimensões de justiça, observa-se que a justiça distributiva foi a primeira a ser utilizada em relações sociais. Esta dimensão de justiça advém diretamente da teoria da equidade e está relacionada aos recursos que os clientes desejam em troca ao que disponibilizaram em um processo de transação social. Segundo a teoria da equidade, em relações sociais, cada uma das partes disponibiliza recursos e recebe resultados da transação, contudo, a troca só é vista como justa quando há a percepção de que os resultados obtidos ultrapassam os recursos disponibilizados (AWASTHI E MEENAKSHI,2013; DEL RÍO-LANZA, VÁZQUEZ-CASIELLES e DÍAZ-MARTÍN, 2009; GOODWIN e ROSS, 1990; OLIVER E SWAN, 1989; SANTOS, 2001; SVARI, 2010; TAX et al., 1998).

Já a justiça processual está relacionada a métodos justos e pacíficos de gerenciar, moderar ou resolver disputas, enfocando, dessa forma, os meios para os quais os fins são alcançados (SIU, ZHANG E YAU, 2013; THIBAUT e WALKER apud SANTOS, 2001). Neste contexto, alguns dos aspectos de maior relevância relacionados à justiça processual em um processo de recuperação de serviços são: a velocidade com que se responde à reclamação feita, a abertura para que o reclamante apresente seu ponto de vista (controle do processo), a facilidade para entrar ou sair do processo, a flexibilidade para adaptação do processo à 
necessidade do reclamante e a aceitação da responsabilidade por parte do prestador de serviços (HILL e BAER, 1994; SANTOS, 2001; TAX et al,. 1998).

Por fim, a justiça interpessoal é avaliada pelo cliente no momento das suas interações com o fornecedor de serviços e envolve a sua percepção acerca da postura do funcionário de serviços (TAX et al., 1998), incluindo a sensibilidade interpessoal do tratamento, ou seja, a capacidade do funcionário tratar os clientes com dignidade e respeito, fornecendo explicações adequadas para o problema percebido no serviço (HA E JANG, 2009). Por este motivo, aspectos como a explicação das causas do problema, a honestidade, a educação/cortesia, a demonstração de esforço na resolução do problema, a empatia (interesse genuíno de entender e resolver o problema do cliente) e um pedido de desculpas de forma sincera são fundamentais para que esta dimensão de justiça seja bem avaliada pelos clientes (ROSCHK e KAISER, 2013; SANTOS, 2001; TAX et al., 1998).

Neste contexto, considerando que a utilização da teoria da justiça a partir da avaliação das suas três dimensões, é extremamente útil para se modelar as percepções de clientes reclamantes após a ocorrência de uma falha de serviços, espera-se que as percepções de justiça distributiva, processual e interpessoal, operacionalizadas a partir de seus principais elementos propostos em trabalhos anteriores, relacionem-se positivamente com a satisfação dos clientes após o gerenciamento de reclamações operacionalizado pela empresa prestadora.

\subsection{Satisfação do consumidor}

O conceito de satisfação representa função central na literatura de serviços e avalia satisfação como a resposta ao contentamento do consumidor, ou seja, uma análise realizada pelo usuário do nível de prazer que o serviço lhe ofereceu (OLIVER, 2014).

A satisfação do consumidor é uma condição almejada por todas as organizações, pois está diretamente relacionada com a lealdade à marca, repetição de compras, comunicação boca a boca positiva, lucratividade e participação de mercado (FARIAS e SANTOS, 2000).

Uma abordagem eficaz para se avaliar a satisfação após a utilização de produtos ou serviços é a desconfirmação de expectativas, que consiste em comparar o desempenho do serviço percebido pelo cliente com as suas expectativas antes da

Revista Produção Online, Florianópolis, SC, v.16, n. 1, p. 126-150, jan./mar. 2016. 
utilização (OLIVER, 1980). Assim, assegurar a entrega do serviço com um desempenho superior às expectativas é importante para empresas fornecedoras de serviço porque quanto maior é o nível de satisfação percebido pelos clientes, maior tende a ser a sua intenção de reutilização do serviço fornecido (OLIVER, 1980).

Ressalta-se que o uso da desconfirmação de expectativas para avaliação da satisfação se mostra adequado nas transações envolvendo bens não duráveis ou de baixo valor, pois, nos casos dos bens duráveis, há um maior envolvimento do cliente no processo de consumo, o que torna o desempenho observado mais importante para a satisfação final do que a desconfirmação das expectativas anteriores ao consumo (CHURCHILL e SURPRENANT, 1982; ROOS; SARTORI e GODOY, 2009).

Neste contexto, o presente estudo avaliou a satisfação do usuário a partir do paradigma da desconfirmação de expectativas, considerando primeiramente a sua robustez, verificada em diversas pesquisas empíricas (ver, por exemplo, BORGES JR e FONSECA, 2002; GOSLING e COELHO, 2014; SANTOS e FERNANDES, 2008), e, adicionalmente, pelo fato do serviço de telefonia celular ser uma transação não durável, por definição, e de baixo envolvimento por parte do usuário (CHURCHILL e SURPRENANT, 1982).

\section{PROCEDIMENTOS METODOLÓGICOS}

Para a operacionalização da pesquisa foi conduzido um estudo de caso (YIN, 2005) no contexto de uma empresa de telefonia celular, que faz parte do setor de telecomunicações brasileiro e que, segundo os órgãos de defesa do consumidor, tem gerado uma grande quantidade de reclamações de clientes em decorrência de falhas de serviços (FOLHA ONLINE, 2013).

A pesquisa teve como unidades de análise os episódios gerados a partir reclamações de 496 clientes que, após terem ligado para o call center da empresa para reclamar de aspectos relacionados ao serviço prestado e/ou solicitar o seu cancelamento, foram atendidos pelos grupos de atendimento personalizado e, a partir de então, passaram por um processo de gerenciamento de reclamações conduzidos pela organização.

Para a condução da pesquisa, foi utilizado um instrumento de coleta de dados contendo 23 questões retratando os construtos analisados e outras 3 questões

Revista Produção Online, Florianópolis, SC, v.16, n. 1, p. 126-150, jan./mar. 2016. 
voltadas para a identificação de dados demográficos dos entrevistados. Na tabela 1 são apresentadas as principais obras e escalas utilizadas para elaboração do questionário.

Tabela 1- Escalas utilizadas no instrumento de coleta de dados.

\begin{tabular}{lcc}
\hline \multicolumn{1}{c}{ CONSTRUTOS } & ESCALA & OBRAS \\
\hline Percepção de Justiça Distributiva & Tipo Likert 5 pontos & $\begin{array}{c}\text { Goodwin e Ross (1990); Deutsch } \\
\text { (1975); Tax et al. (1998); }\end{array}$ \\
& & Santos (2001) \\
Percepção de Justiça Processual & Tipo Likert 5 pontos & Santos (2001); Tax et al. (1998) \\
Percepção de Justiça & Tipo Likert 5 pontos & Bitner et al. (1990); Santos (2001); Tax \\
Interpessoal & et al. (1998) \\
Satisfação dos clientes & Tipo Likert 5 pontos & Oliver (1980); Santos (2001) \\
\hline
\end{tabular}

\section{ANÁLISE DE DADOS}

Para avaliar a confiabilidade ou consistência interna do instrumento de coleta, foi calculado o alfa de cronbach de cada um dos blocos relacionados às percepções de justiça, conforme recomendam Hair et al. (2005). Os autores sugerem um Alfa de Cronbach mínimo de 0,7 para que se garanta a existência de uma boa consistência na escala e, para estudos exploratórios, valores de alfa acima de 0,6.

Neste estudo, observou-se que o instrumento de coleta de dados mostrou ter uma boa consistência interna, com um alfa de cronbach resultante de 0,88 , acima do aceitável segundo Hair et al., (2005).

Já em relação às validades nomológica e convergente dos construtos, foi utilizada a técnica de Análise Fatorial Confirmatória, onde foram analisados: (a) as cargas fatoriais obtidas, (b) os índices de ajustamento do modelo e (c) a indicação de validade convergente, calculada a partir do coeficiente de correlação de Spearman entre os elementos de cada construto, conforme propõem Hair et al. (2005).

Verificou-se que os construtos de justiça interpessoal e processual demonstraram possuir validade nomológica e convergente, com todas as cargas fatoriais altas e correlações significativas. Além disso, o grau de ajustamento de cada modelo real ao seu respectivo modelo teórico também foi aceitável, pois os

Revista Produção Online, Florianópolis, SC, v.16, n. 1, p. 126-150, jan./mar. 2016. 
índices NFI, NNFI, GFI e AGFI demonstraram aderência igual ou maior que 0,96 e os valores de RMS e RMSEA ficaram abaixo de 0,05, máximo recomendado (HAIR et al., 2005).

Todavia, para a justiça distributiva, a AFC gerou uma carga fatorial não significativa para a questão Q23 'O resultado da minha reclamação não parece ter sido tão bom quanto o de outras pessoas que reclamaram de forma parecida'. Este resultado já era esperado, pois a análise de confiabilidade apontou que a presença desta questão reduzia de forma significativa a consistência interna do bloco de questões. Assim optou-se por não utilizar na análise de dados a questão supracitada.

\subsection{Análises univariadas}

As análises univariadas foram realizadas a partir do cálculo das frequências relativas para caracterização da amostra, além da média e do desvio padrão de cada uma das questões avaliadas a partir de escalas do tipo Likert. Cabe ressaltar que a utilização da média para a avaliação de escalas do tipo likert é algo amplamente aceito em trabalhos na área de ciências sociais, onde estas escalas são consideradas como sendo do tipo intervalar para fins de análise estatística (GÜNTHER, 2003).

Avaliando o perfil da amostra, observa-se que 58,9\% dos respondentes são homens, 39,5\% tem entre 20 e 29 anos, 49\% afirmam ter renda de até $R \$ 2.000$ e $49,2 \%$ tem o $3^{\circ}$ grau completo. Além disso, o maior motivo da reclamação dos clientes se concentrou em problemas com a conta telefônica, representando $29,6 \%$ dos casos.

No que concerne à percepção de justiça interpessoal declarada pelos respondentes, observa-se que o elemento melhor avaliado foi o de 'Educação e Cortesia dos atendentes' do call center. Por outro lado, o elemento de pior avaliação na opinião dos respondentes foi o 'Ter recebido desculpas sinceras', possivelmente devido à postura padronizada do atendimento em que não se permite a presença da emoção na figura do atendente.

$\mathrm{Na}$ avaliação dos elementos de justiça processual, o item de melhor desempenho foi 'Ter tido a chance de contar o problema à empresa', com média de

Revista Produção Online, Florianópolis, SC, v.16, n. 1, p. 126-150, jan./mar. 2016. 
4,3 e coeficiente de variação de $28 \%$. Estes resultados já eram esperados, visto que, em call centers, ouvir o que o cliente tem a dizer é uma condição básica do processo de atendimento de ligações. Por outro lado, destacam-se como aspectos de baixo desempenho a velocidade de resposta à reclamação do cliente e a aceitação da responsabilidade pela empresa, com médias de 2,6 e 1,9, respectivamente. Estas baixas médias configuram um cenário crítico para a empresa, visto que estes são dois aspectos de grande relevância para a avaliação da justiça processual (TAX et al., 1998).

Para os elementos de justiça distributiva, observou-se uma tendência de concentração das respostas em torno do valor central. Um ponto a se ressaltar, porém, foi o fato de que, diferentemente das outras duas dimensões de justiça, nenhuma das questões apresentou média superior ou próxima ao nível 4. Isso demonstra que os aspectos relacionados a resultados na empresa analisada podem atuar de forma negativa na percepção de justiça global dos clientes, que tendem a analisá-la à luz de cada uma das suas 3 dimensões de forma simultânea.

Os resultados das três avaliações das dimensões de justiça podem ser observados na tabela 2 .

Tabela 2 - Média, desvio padrão e coeficiente de variação das questões das três dimensões de justiça

\begin{tabular}{|c|c|c|c|}
\hline Questões & Média & Desvio Padrão & Coef. de variação \\
\hline \multicolumn{4}{|l|}{ Justiça Interpessoal } \\
\hline Eles foram honestos comigo & 3,4 & 1,6 & $49 \%$ \\
\hline $\begin{array}{l}\text { Nenhuma razão me foi dada sobre porque o problema } \\
\text { ocorreu }\end{array}$ & 3,1 & 1,8 & $56 \%$ \\
\hline Eles pareceram muito interessados no meu problema. & 3,2 & 1,7 & $51 \%$ \\
\hline Eles foram educados e corteses comigo. & 4,3 & 1,2 & $28 \%$ \\
\hline $\begin{array}{l}\text { Eles se esforçaram bastante para tentar resolver o } \\
\text { problema. }\end{array}$ & 3,1 & 1,6 & $51 \%$ \\
\hline Eu recebi um pedido de desculpas sincero. & 3,0 & 1,8 & $59 \%$ \\
\hline De maneira geral, eu recebi um tratamento pessoal justo. & 3,3 & 1,6 & $48 \%$ \\
\hline \multicolumn{4}{|l|}{ Justiça Processual } \\
\hline Eles responderam rapidamente minha reclamação. & 2,6 & 1,7 & $63 \%$ \\
\hline $\begin{array}{l}\text { A empresa assumiu a responsabilidade pela ocorrência do } \\
\text { problema. }\end{array}$ & 2,9 & 1,8 & $62 \%$ \\
\hline $\begin{array}{l}\text { O acesso à central de atendimento da claro para fazer a } \\
\text { reclamação foi rápido e fácil. }\end{array}$ & 3,0 & 1,7 & $59 \%$ \\
\hline $\begin{array}{l}\text { Eu tive a chance de contar a eles detalhes sobre meu } \\
\text { problema. }\end{array}$ & 4,3 & 1,2 & $28 \%$ \\
\hline $\begin{array}{l}\text { Eles demonstraram flexibilidade na resolução de minha } \\
\text { reclamação. }\end{array}$ & 3,2 & 1,6 & $50 \%$ \\
\hline $\begin{array}{l}\text { De maneira geral, a empresa teve bons procedimentos para } \\
\text { resolver mipha reçlapacão. }\end{array}$ & 3,1 & 1,6 & $52 \%$ \\
\hline \multicolumn{4}{|c|}{ Justiça Distributiva } \\
\hline O resultado final da reclamação foi o esperado. & 2,9 & 1,8 & $62 \%$ \\
\hline $\begin{array}{l}\text { Eu não recebi o que eu pedi. } \\
\text { O resultado da minha reclamação não parece te }\end{array}$ & 2,9 & 1,8 & $63 \%$ \\
\hline bom quanto o de outras pessoas que reclamaram de forma & 2,7 & 1,5 & $53 \%$ \\
\hline
\end{tabular}


Fonte: Os Autores (2015).

Nas questões relacionadas à importância de cada dimensão de justiça, observou-se que a justiça interpessoal obteve a maior média, seguida da justiça processual e da justiça distributiva. A partir da análise individual desses resultados, poder-se-ia supor que o fator decisivo durante o gerenciamento de reclamações é o tratamento que o cliente recebe dos atendentes, conforme pode ser verificado na tabela 3.

Tabela 3 - Média, desvio padrão e coeficiente de variação da importância de cada dimensão de justiça

\begin{tabular}{lccc}
\hline \multicolumn{1}{c}{ Questões } & Média & Desvio Padrão & Coef. de variação \\
\hline Importância do Tratamento Pessoal & 3,6 & 1,5 & $41 \%$ \\
Importância dos Procedimentos & 3,2 & 1,6 & $49 \%$ \\
Importância dos Resultados & 3,1 & 1,7 & $54 \%$ \\
\hline
\end{tabular}

Fonte: Os Autores (2015).

Já em relação à satisfação, observa-se que 0 item relacionado a este construto teve um desempenho relativamente baixo, com média de 2,8, sobretudo quando comparado com as percepções gerais de justiça cujos valores variaram de 3 a 3,3. Este resultado pode ser verificado na tabela 4.

Tabela 4 - Média, desvio padrão e coeficiente de variação da importância de cada dimensão de justiça

\begin{tabular}{lccc}
\hline \multicolumn{1}{c}{ Questões } & Média & Desvio Padrão & Coef. de variação \\
\hline $\begin{array}{l}\text { A organização fez tudo o que eu esperava para resolver } \\
\text { o meu problema (atendeu às expectativas). }\end{array}$ & 2,8 & 1,7 & $61 \%$ \\
\hline
\end{tabular}

Fonte: Os Autores (2015)

No próximo tópico serão apresentados os resultados da terceira etapa de análise, onde foram utilizadas técnicas estatísticas multivariadas com o objetivo de se levantar as relações entre os construtos e, a partir daí, compará-las com os resultados das análises univariadas.

Revista Produção Online, Florianópolis, SC, v.16, n. 1, p. 126-150, jan./mar. 2016. 


\subsection{Análises multivariadas}

Após condução dos testes de hipótese para avaliação da normalidade da amostra, observou-se que, para $p<0,001$, todas as variáveis apresentaram distribuições não-paramétricas, o que indicou a necessidade de se escolher técnicas estatísticas multivariadas específicas para dados não paramétricos. Neste sentido, considerando a característica não-paramétrica das variáveis, foi escolhida a técnica de Regressão Logística, que não tem como pressuposto básico a característica paramétrica das distribuições das variáveis (HAIR et al., 2005).

$\mathrm{Na}$ operacionalização da regressão, foi escolhido o método Stepwise Backward $L R$, utilizando uma transformação das escalas tipo Likert de 5 ou 10 pontos para escalas binárias, com as respostas possíveis sendo 0 ou 1. Este tipo de transformação foi feito para permitir a visualização direta do efeito que a presença de determinada variável independente gera na variável dependente, denominado de Odds Ratio ou razão de chances (TABACHNICK e FIDELL, 2001).

Para a avaliação da justiça interpessoal, foi gerado um modelo logístico de variáveis binárias, composto pela variável dependente 'Percepção de justiça no tratamento recebido' e pelos seus elementos individuais como variáveis independentes.

Neste modelo, que apresentou um bom poder preditivo, com acerto médio de $78,1 \%$, o elemento que mostrou ter maior influência foi 'Ter recebido um pedido de desculpas sincero', cujo Odds Ratio, calculado a partir da exponencial do coeficiente de regressão $B$, foi de 4,77. Este resultado significa que, permanecendo constantes todos os demais elementos, a afirmação de que recebeu um pedido de desculpas sincero aumenta em 4,77 vezes as chances do cliente considerar que obteve um tratamento pessoal justo.

Além disso, cabe ressaltar que a presença dos motivos de falha relacionados à Cobertura/Rede e a outros tipos menos freqüentes, possivelmente se deu devido à facilidade com que os atendentes conseguem tratar estas reclamações, pois, normalmente, não envolvem questões financeiras. Dessa forma, espera-se que os clientes avaliem mais positivamente a postura dos atendentes em situações de atendimentos de baixa complexidade. 
Outro ponto relevante se concentra na ausência da variável relacionada à Gravidade da falha no modelo final. Esta variável não influencia a percepção de justiça interpessoal, pois, por mais insatisfeitos que estejam os clientes, estes parecem não atribuir a culpa pela falha crítica ao atendente, separando, assim, a figura do funcionário de serviços da empresa prestadora. Os coeficientes do modelo de justiça interpessoal podem ser verificados na tabela 5.

Tabela 5 - Coeficientes do modelo de regressão de justiça interpessoal

\begin{tabular}{lcccccc}
\hline Variáveis & B & S.E. & Wald & GL & Sig. & Exp(B) \\
\hline Falha de Cobertura e Rede & 1,036 & 0,304 & 11,620 & 1 & 0,001 & 2,82 \\
Outros tipos de Falhas & 0,969 & 0,361 & 7,215 & 1 & 0,007 & 2,64 \\
Honestidade & 1,183 & 0,250 & 22,301 & 1 & 0,000 & 3,26 \\
Interesse pelo Problema & 1,076 & 0,278 & 14,964 & 1 & 0,000 & 2,93 \\
Educação/Cortesia & 0,760 & 0,328 & 5,359 & 1 & 0,021 & 2,14 \\
Esforço & 0,928 & 0,284 & 10,654 & 1 & 0,001 & 2,53 \\
Pedido de Desculpas & 1,563 & 0,253 & 38,036 & 1 & 0,000 & 4,77 \\
Constante & $-3,036$ & 0,359 & 71,571 & 1 & 0,000 & 0,05 \\
\hline
\end{tabular}

Fonte: Os Autores (2015).

Para o construto percepção de justiça processual, chegou-se a um modelo logístico após 8 iterações. Neste modelo, observa-se a ausência do elemento 'Facilidade de acesso ao call center', de forma similar aos resultados encontrados nas análises anteriores onde o item não apresentou correlação alta com a percepção global de justiça processual. Uma possível explicação para este resultado se concentra no item 'Facilidade de Acesso' não ser entendido como um procedimento pelos clientes, mas sim por algo inerente à infra-estrutura da empresa.

Vale ressaltar, ainda, a grande importância dada aos elementos 'Assumir Responsabilidade' e 'Flexibilidade' que apresentaram Odds Ratios de 5,29 e 6,83, respectivamente. Esses resultados indicam sérios problemas quando comparados com os resultados obtidos nas análises univariadas, nas quais ambos os itens obtiveram avaliações médias baixas.

Para este modelo, observa-se, ainda, uma forte influência negativa da Gravidade da falha, em que o Odds Ratio foi de 0,39. Este resultado aponta que, 
quando a falha é considerada como grave, as chances do cliente perceber o processo como justo caem mais que a metade, indicando a importância que deve ser dada para os demais elementos de justiça em situações de falhas graves, de modo a reduzir os efeitos da magnitude da falha para os clientes. Os coeficientes do modelo logístico de justiça processual, que apresentou um poder preditivo de $79,6 \%$ de acertos, podem ser verificados na tabela 6 .

Tabela 6 - Coeficientes do modelo de regressão de justiça processual.

\begin{tabular}{lcccccc}
\hline Variáveis & B & S.E. & Wald & GL & Sig. & $\operatorname{Exp}(\mathbf{B})$ \\
\hline Gravidade da Falha & $-0,946$ & 0,267 & 12,537 & 1 & 0,000 & 0,39 \\
Falha de Cobertura e Rede & 0,491 & 0,287 & 2,912 & 1 & 0,088 & 1,63 \\
Resposta rápida & 0,572 & 0,260 & 4,824 & 1 & 0,028 & 1,77 \\
Assumir responsabilidade & 1,667 & 0,250 & 44,531 & 1 & 0,000 & 5,29 \\
Chance de contar o Probl. & 0,917 & 0,370 & 6,143 & 1 & 0,013 & 2,50 \\
Flexibilidade & 1,922 & 0,248 & 59,872 & 1 & 0,000 & 6,83 \\
Constante & $-2,344$ & 0,442 & 28,118 & 1 & 0,000 & 0,10 \\
\hline
\end{tabular}

Fonte: Os Autores (2015).

No modelo Logístico calculado para a justiça distributiva, observa-se a forte influência do elemento 'Receber resultados esperados'. Um alto valor para a influência deste item já era esperado, dada a alta correlação encontrada entre a percepção global de justiça e este elemento.

Observou-se, ainda, que a variável relacionada a 'Não ter recebido o que foi pedido' não se mostrou significativa para o modelo de regressão. Da mesma forma, este resultado não surpreende tendo em vista a baixa intensidade da correlação identificada entre ela e a percepção global de justiça distributiva. Todos os coeficientes do modelo logístico de justiça distributiva podem ser verificados na tabela 7

Tabela 7 - Coeficientes do modelo de regressão de justiça distributiva.

\begin{tabular}{|c|c|c|c|c|c|c|}
\hline Variáveis & $\overline{\mathbf{B}}$ & S.E. & Wald & GL & Sig. & $\operatorname{Exp}(B)$ \\
\hline Gravidade da Falha & $-0,510$ & 0,257 & 3,923 & 1 & 0,048 & 0,60 \\
\hline Ter renda Alta & $-0,617$ & 0,245 & 6,355 & 1 & 0,012 & 0,54 \\
\hline Resultados esperados & 2,917 & 0,244 & 143,307 & 1 & 0,000 & 18,49 \\
\hline Ter carência & $-0,422$ & 0,238 & 3,136 & 1 & 0,077 & 0,66 \\
\hline Constante & $-0,824$ & 0,280 & 8,667 & 1 & 0,003 & 0,44 \\
\hline
\end{tabular}


Fonte: Os Autores (2015)

Para este modelo, além da influência negativa da gravidade da falha, quando o perfil do respondente indica uma renda igual ou superior $R \$ 2.000$, as chances de haver a percepção de obtenção de um resultado justo caem quase que pela metade. Este resultado possivelmente se dá devido ao fato de que, para pessoas com maiores rendas, as expectativas quanto aos resultados financeiros tendem a ser maiores, reduzindo a percepção de recebimento de resultados justos.

Um outro ponto relevante é a influência da carência contratual dos clientes. Possivelmente a presença deste fator, se dá porque independente do resultado recebido, o cliente não pode solicitar o cancelamento do serviço sem que pague uma multa contratual, levando-o a considerar o resultado como injusto. Por fim, em relação ao poder preditivo, observa-se que o modelo apresentou o maior percentual de acerto dentre as três dimensões de justiça, com 81,8\% de classificações corretas.

Objetivando avaliar a relação entre cada uma das percepções globais de justiça e a satisfação após o gerenciamento de reclamações, foi elaborado um novo modelo de regressão logística em que, além das dimensões de justiça, foram inicialmente inclusos aspectos relacionados às falhas, como tipologia e gravidade, além das características demográficas dos clientes.

Como resultado, observou-se que, após 11 iterações, apenas as dimensões de justiça distributiva e processual se mostraram significativas. Para estas duas dimensões foram encontrados altos índices de Odds Ratio, sendo que a justiça processual obteve o maior coeficiente: 5,53 .

Comparando estes resultados com as análises univariadas, verifica-se que a justiça interpessoal, mesmo tendo sido apontada como a de maior relevância dentre as três dimensões, não mostrou relação significativa com a satisfação, o que indica que, apesar de considerar importante o tratamento pessoal, este fator não é determinante para a satisfação dos clientes. Assim, os fatores que efetivamente antecedem a satisfação dos clientes são a presença de bons procedimentos e de resultados justos.

Observa-se, ainda, que, quando a falha é apontada como grave, as chances de que o cliente fique satisfeito após o gerenciamento de reclamações caem pela metade. Este resultado reforça a necessidade de que as dimensões de justiça sejam

Revista Produção Online, Florianópolis, SC, v.16, n. 1, p. 126-150, jan./mar. 2016. 
devidamente gerenciadas para que, mesmo em situações de falhas graves, a sua satisfação possa ser obtida.

Por fim, outro fator que merece atenção é a presença do elemento falha de Cobrança no modelo final, com um baixo Odds Ratio de 0,36. Esse resultado demonstra que, para problemas de cobrança, as chances de se confirmar as expectativas dos clientes são muito baixas. Isso se dá, possivelmente, devido ao impacto que este tipo de falha gera, pois, muitas vezes, envolve o envio do CPF do cliente para os órgãos de proteção de crédito como o SPC e o SERASA. Os coeficientes de regressão podem ser verificados na tabela 8.

Tabela 8 - Coeficientes do modelo completo de regressão para a satisfação.

\begin{tabular}{lcccccc}
\hline Variáveis & B & S.E. & Wald & GL & Sig. & Exp(B) \\
\hline Procedimentos justos & 1,710 & 0,251 & 46,424 & 1 & 0,000 & 5,53 \\
Resultados justos & 1,643 & 0,243 & 45,654 & 1 & 0,000 & 5,17 \\
Gravidade da falha & $-0,720$ & 0,258 & 7,754 & 1 & 0,005 & 0,49 \\
Falhas de Cobrança & $-1,014$ & 0,549 & 3,416 & 1 & 0,065 & 0,36 \\
Constante & $-1,590$ & 0,286 & 30,886 & 1 & 0,000 & 0,20 \\
\hline
\end{tabular}

Fonte: Os Autores (2015).

Todavia, com o intuito, de entender o porquê da ausência da justiça interpessoal do modelo calculado, a análise de regressão logística foi novamente conduzida, porém agora considerando apenas as situações de gravidade de falhas baixa (0) indicada pelos respondentes. Com esta nova análise, observou-se que, diferentemente dos resultados obtidos anteriormente, a dimensão de justiça interpessoal obtida foi significativa, com um Odds Ratio de 2,49. Pode-se concluir a partir deste resultado que, mesmo ativando apenas um dos sentidos humanos, em situações de baixo impacto das falhas, a forma pela qual o funcionário trata o cliente se torna relevante, pois a satisfação tende a ser obtida mais facilmente, sobretudo com a percepção de que o tratamento pessoal recebido foi bom. Os novos coeficientes do modelo discutido podem ser observados na tabela 9.

Tabela 9 - Coeficientes do modelo alternativo de regressão para a satisfação em situações de falhas leves

\begin{tabular}{|c|c|c|c|c|c|c|}
\hline Variáveis & $\overline{\mathbf{B}}$ & S.E. & Wald & $\overline{G L}$ & Sig. & $\operatorname{Exp}(B)$ \\
\hline Tratamento justo & 0,911 & 0,498 & 3,351 & 1 & 0,067 & 2,49 \\
\hline $\begin{array}{l}\text { Procedimentos justos } \\
\text { Resultadistâ juoductos justos }\end{array}$ & $\begin{array}{l}\text { 2,27,1 } \\
\text { riąnóp }\end{array}$ & ${ }_{0,530}^{0,530}$ & $\begin{array}{l}18,327 \\
, 7,4,41 p\end{array}$ & $1-15$ & $\begin{array}{l}0,000 \\
n, \text { mar } \\
0,006\end{array}$ & 19,69 \\
\hline Falhas de Conta & $-0,890$ & 0,538 & 2,741 & 1 & 0,098 & 0,41 \\
\hline Falhas de Cobrança & $-1,683$ & 0,867 & 3,770 & 1 & 0,052 & 0,19 \\
\hline Constante & $-2,167$ & 0,534 & 16,436 & 1 & 0,000 & 0,11 \\
\hline
\end{tabular}


Fonte: Os Autores (2015).

\section{CONCLUSÕES}

Este estudo avaliou, dentro do contexto de prestação de serviços de uma empresa de telefonia celular, as percepções de justiça dos clientes após um episódio de gerenciamento de reclamações e a sua relação com a satisfação transacional após este processo.

Inicialmente, ao avaliar os resultados individuais de cada dimensão de justiça, observa-se que, apesar do elemento de justiça interpessoal 'Educação e Cortesia dos Atendentes' ter tido a melhor avaliação individual por parte dos respondentes, a sua participação na percepção geral de justiça interpessoal foi a de menor relevância em comparação com os demais elementos. Este é um resultado de grande importância, pois, apesar de aspectos relacionados à educação e cortesia no atendimento serem as competências mais fortemente trabalhadas em operações de call centers, este efetivamente não é o item de maior importância para a percepção de justiça interpessoal.

Em contrapartida, verificou-se, que um 'Pedido de desculpas sincero' mostrou ser o elemento mais importante de justiça interpessoal, com o dobro da influência do quesito 'Educação e Cortesia', apesar da avaliação individual deste item por parte dos respondentes, ter obtido a menor média. A partir destes resultados, verifica-se que a empresa deve reorientar seus esforços de treinamento no sentido de melhorar o seu atendimento a clientes com vistas aos itens que demonstraram ser os mais influentes antecedentes da percepção de justiça interpessoal, fugindo, assim, do 'lugar comum' da maioria dos call centers que tende a priorizar o elemento de educação e cortesia dos atendentes.

Já em relação aos elementos de justiça processual, da mesma forma que ocorreu com a justiça interpessoal, foi verificado que os itens 'Flexibilidade' e 'Assumir responsabilidade', mal-avaliados individualmente pelos respondentes, mostraram ter grande influência na percepção geral de justiça. Em contrapartida, o

Revista Produção Online, Florianópolis, SC, v.16, n. 1, p. 126-150, jan./mar. 2016. 
item 'Chance de contar o problema', que obteve a melhor média de avaliação individual, mostrou ter um efeito na percepção de justiça processual que, apesar de ser significativo, é relativamente baixo. Isso demonstra, novamente, a necessidade de se reavaliar a importância dada a cada aspecto do processo de atendimento a clientes reclamantes na operação analisada, de modo a se garantir que os elementos de maior relevância que antecedem a percepção de justiça processual sejam gerenciados de forma mais eficaz.

Considerando que em estudos anteriores (ver, por exemplo, SANTOS e FERNANDES, 2008; TAX et al., 1998) não tem sido encontrados padrões recorrentes no que concerne à importância dos diversos elementos de justiça dentro do contexto de cada dimensão, estes resultados apontam para uma característica específica do caso avaliado e, por este motivo, só podem ser utilizados para avaliar contextos de naturezas correlatas.

Analisando a influência das dimensões de justiça na satisfação após o gerenciamento de reclamações, observa-se que as dimensões de justiça processual e distributiva mostraram ter influência significativa, apesar de não ter sido observada a influência significativa da justiça interpessoal na satisfação dos clientes.

Um dos possíveis motivos para a ausência da justiça interpessoal do modelo final é que, para atendimento em call centers, o fato de não existir um contato facea-face com o cliente faz com que a importância deste fator para a satisfação dos clientes seja reduzida, pois, nestes casos, o comportamento do funcionário de serviços ativa apenas um dos sentidos do cliente: a audição. Assim, o fato de este estudo ter sido conduzido em um contexto diferente daqueles utilizados em outras pesquisas, que avaliaram relações de prestação de serviços sem necessariamente 0 cliente ter utilizado um call center para reclamar, pode ser visto como um dos motivos desta ausência.

Além disso, ao se avaliar a ordem de importância e significância da influência de cada dimensão de justiça na satisfação dos clientes, observa-se que estudos anteriores não tem identificado um padrão único. Verifica-se, por exemplo, que nos estudos de Tax et al. (1998) e Blodgett et al. (1997), o efeito encontrado da justiça interpessoal na satisfação foi superior aos das outras dimensões de justiça. Já nos estudos de Goodwin e Ross (1992) e Smith et al. (1999), a dimensão de justiça de maior relevância para a satisfação foi a distributiva, enquanto que Santos (2001)

Revista Produção Online, Florianópolis, SC, v.16, n. 1, p. 126-150, jan./mar. 2016. 
sequer identificou relação significativa entre a percepção de justiça processual e a satisfação. Assim, este trabalho corrobora a idéia de que a influência da percepção dos diversos tipos de justiça na satisfação do cliente varia de acordo com 0 segmento estudado (RUYTER e WETZELS, 2000).

Um ponto que merece destaque se concentra na relevância da variável 'Gravidade das falhas' enquanto antecedente da satisfação. A presença deste fator aponta que, no contexto estudado, quando os clientes identificam as falhas como graves, as chances destes ficarem satisfeitos após o gerenciamento de reclamações caem pela metade. Este resultado é similar ao encontrado por Miller et al. (1999), apontando que mesmo que o call center conduza um excelente gerenciamento de reclamações, para situações de falhas graves, possivelmente o cliente sairá insatisfeito ao final do processo de atendimento.

Pode-se concluir, assim, que os processos de gerenciamento de reclamações são fundamentais para o desenho e projeto de serviços e redes de serviços, sobretudo para contextos de operação com características de grande incidência de falhas de serviços, como é o de Telecom. Cabe ressaltar, contudo, que estes processos, apesar de serem importantes, não são as panacéias que vão resolver todos os problemas criados pela empresa, fazendo com que sejam necessárias revisões de processos no intuito de reduzir a incidência de falhas de serviços, especialmente das consideradas graves. Neste contexto, estas ações voltadas podem reduzir a quantidade de reclamações dos clientes e, por conseguinte, a insatisfação por elas gerada.

Por fim, verifica-se que uma implicação gerencial importante se concentra na necessidade de se cruzar as informações das estatísticas univariadas, normalmente obtidas a partir de pesquisas de satisfação de clientes com aquelas obtidas a partir de técnicas multivariadas, como a regressão logística. Dessa forma, os gerentes responsáveis pelos processos de atendimento da empresa poderão verificar claras oportunidades de melhorias no que concerne à priorização de ações que efetivamente atuem no sentido de melhorar a percepção de cada uma das três dimensões de justiça.

\section{REFERÊNCIAS}


ALMEIDA, L. O. A.; TOLEDO, G. L. Retenção De Clientes: recuperação de falhas de serviços. In: Anais do VI SEMEAD, São Paulo, 2003. Anais...FEA-USP, 2003 http://dx.doi.org/10.5195/emaj.2014.45

ASSEFA, E. S. The effects of Justice Oriented Service Recovery on Customer Satisfaction and Loyalty in Retail Banks in Ethiopia. Emerging Markets Journal, v. 4, p. 49-58. 2014 http://dx.doi.org/10.5195/emaj.2014.45

AWASTHI, A. K. RAWANI, M. .Effect of Organisational Responses on Customer's Perceived Justice: Moderating Role of Causal Attributions. Journal of Marketing \& Communication, v. 9, p62-65. 2013

BATTAGLIA, D.; BORCHARDT, M.. Análise do processo de recuperação de serviços a partir das reclamações dos clientes: estudo de caso em três organizações. Revista Produção, v. 20 , n. 3, p. 455-470, 2010. http://dx.doi.org/10.1590/S0103-65132010005000028

BORGES JR, A. A.; FONSECA, M. J. O uso da pesquisa de satisfação do consumidor como instrumento de política pública: o potencial de uso no caso do transporte coletivo de Porto Alegre. Revista Interdisciplinar de Marketing, v. 1, n. 3, p. 38-50, 2002.

BRATA, H.; JULIANA, L. Performance-based reward systems and perceived justice: a case of motorbike dealer in Pontianak. International Journal of Business \& Society, v. 15, p 195-214. 20p, 2014. http://dx.doi.org/10.5539/ijbm.v6n12p270

BERGER, C.; BLAUTH, R.; BOGER, D.; et al. Kano's methods for understanding customerdefined quality. Center for Quality Management Journal, Massachusetts, v. 2, n. 4, p. 335, fall 1993.

BITNER, M. J.; BOOMS, B. H.; MOHR, L. A. Critical service encounters: the employee's viewpoint. Journal of marketing, v. 58, n. 4, p. 95-105, 1994.

http://dx.doi.org10.2307/1251919

BITNER, M. J.; BOOMS, B. H.; TETREAULT, M. S. The Service Encounter: Diagnosing Favorable and Unfavorable Incidents. Journal of Marketing, v. 54, p. 71-84, 1990.

http://dx.doi.org/10.2307/1252174

BITNER, M. J.; BROWN, S.; MEUTER, M. Technology infusion in service encounters. Academy of Marketing Science Journal, v. 28, n.1, p. 138-149, 2000.

http://dx.doi.org/10.1177/0092070300281013

BLODGETT, J. G.; HILL, D.; TAX, S. S. The effects of distributive, procedural, and interpessoal justice on postcomplaint behavior. Journal of Retailing, v. 73, n. 2, p. 185210,1997. http://dx.doi.org/10.1016/S0022-4359(97)90003-8

BOSHOFF, C. An experimental study of service recovery options. International Journal of Service Industry Management, v. 8, n. 2, p. 110-130, 1997.

http://dx.doi.org/10.1108/09564239710166245

CHEN, Houn-Gee et al. The impact of financial services quality and fairness on customer satisfaction. Managing Service Quality, v. 22, n. 4, p. 399-421, 2012.

http://dx.doi.org/10.1108/09604521211253496

CHURCHILL, G. A. Jr. Marketing Research: methodological foundations. 7. ed. Orlando: The Dryden Press, 1999.

Revista Produção Online, Florianópolis, SC, v.16, n. 1, p. 126-150, jan./mar. 2016. 
CHURCHILL, G.A., SURPRENANT, C. An Investigation into the Determinants of Customer Satisfaction. Journal of Marketing Research, v. 19, n. 4, p. 491-504, 1982.

http://dx.doi.org/10.2307/3151722

DEL RÍO-LANZA, A.; VÁZQUEZ-CASIELLES, R.; DÍAZ-MARTÍN, A. Satisfaction with service recovery: Perceived justice and emotional responses. Journal of Business Research, v. 62, n. 8, p. 775-781, 2009. http://dx.doi.org/10.1016/j.jbusres.2008.09.015

FARIAS, S. A. de, \& SANTOS, R. C. Modelagem de equações estruturais e satisfação do consumidor: uma investigação teórica e prática. Revista de Administração Contemporânea (RAC), v. 4, n. 3, p. 107-132, 2000. http://dx.doi.org/10.1590/S1415$\underline{65552000000300007}$

FOLHA ONLINE. Telefonia lidera ranking de reclamações de consumidores no país. Disponível em: <http://www1.folha.uol.com.br/mercado/2013/11/1375148-clientes-da-clarosao-os-que-mais-reclamam-do-servico-pelo-call-center-diz-anatel.shtml> Acesso em: 10 de Dezembro. 2014.

FREITAS, A. L. A QUALIDADE EM SERVIÇOS NO CONTEXTO DA COMPETITIVIDADE. Revista Produção Online, Florianópolis, v. 5, n. 1, jun. 2005. ISSN 16761901. http://dx.doi.org/10.14488/1676-1901.v5i1.321

GILLILAND, S.W. The perceived fairness of selection systems: An organizational justice perspective. The Academy of Management Review, vol. 18, n. 4, p. 694-734, 1993. http://dx.doi.org/10.5465/AMR.1993.9402210155

GOSLING, M.; COELHO, M.; RESENDE, M. P. D. L.. Qualidade Percebida e Intenções Comportamentais de visitantes em Museus: uma proposta de modelo. Turismo-Visão e Ação, v. 16, n. 3, p. 656, 2014. http://dx.doi.org/10.14210/rtva.v16n3.p656-689

GOODWIN, C.; ROSS, I. Consumer Evaluations of Responses to Complaints: What's Fair and Why. The Journal of Consumer Marketing, v. 7, n. 2, p. 39-47, 1990.

http://dx.doi.org/10.1108/EUM0000000002575

GOODWIN, C.; ROSS, I. Consumer Responses to service failures: Influence of procedural and interactional fairness perceptions. Journal of Business research, v. 25, n. 2, p. 149-63, 1992. http://dx.doi.org10.1016/0148-2963(92)90014-3

GILLY, M. C.; GELB, B. Post-purchase consumer processes and the complaining consumer. Journal of Consumer Research, v. 9, p. 323-328, 1982.

http://dx.doi.org/10.1086/208927

GÜNTHER, H. Como Elaborar um Questionário. Série: Planejamento de Pesquisa nas Ciências Sociais, n. 1. Brasília, DF: UnB, Laboratório de Psicologia Ambiental, 2003.

HA, J.; JANG, S. Perceived justice in service recovery and behavioral intentions: the role of relationship quality. , International Journal of Hospitality Management , v. 28, n. 3, p. $319-27,2009$. http://dx.doi.org/10.1016/j.ijhm.2008.12.001

HAIR Jr., J.; ANDERSON, R. E.; TATHAM, R. L.; BLACK, W. C. Análise Multivariada de Dados. 5. ed. Porto Alegre: Bookman, 2005. 
HALL, A.L; RIST, RC., Integrating Multiple Qualitative Research Methods. Psychology and Marketing, v. 16, n. 4, p.291-304, 1999.

http://dx.doi.org/10.1002/(SICI)1520-6793(199907)16:4<291::AID-MAR2>3.0.CO;2-\#

HART, W. L.; HESKETT, J. L.; SASSER, W. E. The Profitable Art of Service Recovery. Harvard Business Review, v. 68, n. 4, p. 148-156, 1990.

http://dx.doi.org/10.1225/90407

HILL, D. J.; BAER, R. Customers complain - business make excuses: the effects of linkage and valence. Advances in Consumer Research, v. 21, p. 399-405, 1994.

HOFFMAN, K D.; KELLEY, S. W; ROTALSKY. Tracking service failures and employee recovery efforts. Journal of service marketing. v. 9, n. 2, 1995.

http://dx.doi.org/10.1108/08876049510086017

KANO N.; SERAKU N.; TAKAHASHI F.; TSUJI S. Attractive quality vs must be quality. Journal of the Japanese Society for Quality Control, v. 14, n. 2, p. 39-48, 1984

KOMUNDA, M.; OSARENKHOE, A. Remedy or cure for service failure?: Effects of service recovery on customer satisfaction and loyalty.Business Process Management Journal, v. 18, n. 1, p. 82-103, 2012. http://dx.doi.org/10.1108/14637151211215028

LIN, H.; WANG, Y.; CHANG, L. Consumer responses to online retailer's service recovery after a service failure: a perspective of justice theory. Managing Service Quality, v. 21, n. 5, p. 511-534, 2011. http://dx.doi.org/10.1108/09604521111159807

KELLY, S.; DAVIS, M.; HOFFMAN, K. D. A typology of retail failures and recoveries. Journal of Retailing, n. 69, p. 429-452. 1993. http://dx.doi.org/10.1016/0022-4359(93)90016-C

LOVELOCK, C. H., WRIGHT, L. Serviços: marketing e gestão. São Paulo: Saraiva, 2002.

LOVELOCK, C.; WIRTZ, J.; HEMZO, M. Marketing de serviços: pessoas, tecnologia e estratégia. São Paulo: Pearson, 2011.

MALHOTRA, N. K et al. Introdução à Pesquisa de Marketing. São Paulo: Pearson, 2005.

MATTILA, A. S. The impact of relationship type on customer loyalty in a context of service failures. Journal of Service Research, v. 4, n.2, p. 90-101, 2001.

http://dx.doi.org/10.1177/109467050142002

MATZLER, K.; HINTERHUBER, H. H.; BAILON, F.; SAUERWEIN, E. How to delight your customers. Journal of Product \& Brand Management, Santa Barbara, v. 5, n. 2, p. 6-17, mar. 1996. http://dx.doi.org/10.1108/10610429610119469

MICHEL, S. Analyzing service failures and recoveries: a process approach. International Journal of Service Industry Management, v. 12, n. 1, p. 20-33, 2001.

http://dx.doi.org/10.1108/09564230110382754

MILLER, J. L.; CRAIGHEAD, C. W.; KARWAN, K. R. Service recovery: a Framework and empirical investigation. Journal of Operations Management, v. 18, p. 387-400, 2000. http://dx.doi.org10.1016/S0272-6963(00)00032-2

OLIVER, R. L. A Cognitive Model of the antecedents and consequences of satisfaction decisions. Journal of Marketing Research, v. 17, p. 406-469, 1980.

Revista Produção Online, Florianópolis, SC, v.16, n. 1, p. 126-150, jan./mar. 2016. 
OLIVER, R. L.; SWAN, J. E. Consumer Perceptions of Interpersonal Equity and Satisfaction in Transactions: A Field Survey Approach. Journal of Marketing, v. 53, n. 2, p. 21-35, 1989. http://dx.doi.org/10.2307/125141

OLIVER, R. L. Whence Consumer Loyalty? Journal of Marketing, v. 63, n. 4, p. 33-44, 1999. http://dx.doi.org/10.2307/1252099

OLIVER, R. L. Satisfaction: A behavioral perspective on the consumer. Routledge, 2014.

OZGEN, O.; KURT, S. D. Pre-recovery and post-recovery emotions in the service context: a preliminary study. Managing Service Quality, v. 22, n. 6, p. 592-605, 2012.

http://dx.doi.org/10.1108/09604521211287561

ROSSI, C. ; SLONGO, L; A. Pesquisa de Satisfação de Clientes: o Estado-da-Arte e Proposição de um Método Brasileiro. RAC - Revista de Administração Contemporânea, v. 2, n. 1, p. 101-125, 1998.http://dx.doi.org/10.1590/S1415-65551998000100007

ROSCHK, H.; KAISER, S. The nature of an apology: An experimental study on how to apologize after a service failure. Marketing Letters, v. 24, n. 3, p. 293-309, 2013.

http://dx.doi.org/10.1007/s11002-012-9218-x

ROOS, C; SARTORI, S; GODOY, L.P; Modelo de Kano para a identificação de atributos capazes de superar as expectativas do cliente; Revista Produção Online, v.9, n. 2, p. 536550, set. de 2009. http://dx.doi.org/10.14488/1676-1901.v9i3.186

RUYTER, K.; WETZELS, M. Customer equity considerations in service recovery: A crossindustry perspective. International Journal of Service Industry Management, v. 11, n.1, p. 91-108, 2000. http://dx.doi.org/10.1108/09564230010310303

SANTOS, C. P. O impacto do gerenciamento de reclamações na confiança e lealdade do consumidor, no contexto de trocas relacionais de serviços: construção e teste de um modelo teórico. 252 f. Tese (Doutorado) - Universidade Federal do Rio Grande do Sul, Porto Alegre, 2001

SANTOS, C. P.; FERNANDES, D. V. D. H.. A recuperação de serviços como ferramenta de relacionamento: seu impacto na confiança e lealdade dos clientes. RAE - Revista da Administração de Empresas (Impresso), v. 48, p. 10-24, 2008.

http://dx.doi.org/10.1590/S0034-75902008000100002

SVARI, S.; SVENSSON, G.; SLÅTTEN, T.; EDVARDSSON, B. A DIP-construct of perceived justice in negative service encounters and complaint handling in the Norwegian tourism industry. Managing Service Quality, v. 20, n. 1, p. 26-45, 2010

http://dx.doi.org/10.1108/09604521011011612

SIU N.; ZHANG T.; YAU.C.Y. The Roles of Justice and Customer Satisfaction in Customer Retention: A Lesson from Service Recovery. Journal of Business Ethics. v. 114, p 675686. http://dx.doi.org/10.1007/s10551-013-1713-3

SILVA, M.; LOPES, E. A influência da justiça nos processos de recuperação de falhas no varejo. RAC- Revista de Administração Contemporânea., Curitiba , v. 18, n. 1, p. 3758, 2014. http://dx.doi.org/10.1590/S1415-65552014000100004

Revista Produção Online, Florianópolis, SC, v.16, n. 1, p. 126-150, jan./mar. 2016. 
SLACK, N.; CHAMBERS, S.; HARLAND, C. Administração da produção. São Paulo: Atlas, 1996.

SMITH, A. K.; BOLTON, R. N.; WAGNER, J. A model of customer satisfaction with service encounters involving failure and recovery. Journal of Marketing Research, v. 36, n. 3, p. 356-372, 1999. http://dx.doi.org/10.2307/3152082

SWANSON, S. R.; KELLEY, S. W. Service recovery attributions and word-of-mouth intentions. European Journal of Marketing. Bradford. v.35, n.1, p. 194-211, 2001. http://dx.doi.org/10.1108/03090560110363463

TABACHNICK, B.G.; FIDELL, L.S. Using Multivariate Statistics. 4th ed. Boston: Allyn and Bacon, 2001.

TAX, S.; BROWN, S.; CHANDRASHEKARAN, M. Customer Evaluations of Service Complaint Experiences: Implications for Relationship Marketing. Journal of Marketing, $v$. 62, n. 2, p. 60-76, 1998. http://dx.doi.org/10.2307/1252161

TSAI, C.; YANG, Y.; CHENG, Y.. Does relationship matter?-Customers' response to service failure. Managing Service Quality, v. 24, n. 2, p. 139-159, 2014.

http://dx.doi.org/10.1108/MSQ-06-2013-0113

WANG, K.; HSU, L.; CHIH, W. Retaining customers after service failure recoveries: A contingency model. Managing Service Quality, v. 24, n. 4, p. 1-1, 2014.

http://dx.doi.org/10.1108/MSQ-11-2013-0251

WAQAS, M.; ALI, H.; KHAN, M. A. An investigation of effects of justice recovery dimensions on students' satisfaction with service recovery in higher education environment. International Review on Public and Nonprofit Marketing, v. 11, n. 3, p. 263284, 2014. http://dx.doi.org/10.1007/s12208-014-0120-5

WEBSTER C.; SUNDARAM, D. S. Service consumption criticality in failure recovery. Journal of Business Research, v. 41, p. 153-158, 1998.

http://dx.doi.org/10.1016/S0148-2963(97)00004-0

YIN, R. K. Estudo de caso: planejamento e métodos. 3. ed. Porto Alegre: Bookman, 2005.

ZEITHAML, V.; BERRY, L.; PARASURAMAN, A. The nature and determinants of customer expectations of service. Journal of the Academy of Marketing Science, v. 21, n. 1, p. 112, 1993. http://dx.doi.org/10.1177/0092070393211001

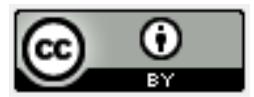

Artigo recebido em 08/04/2015 e aceito para publicação em 27/07/2015

DOI: $\underline{\text { http://dx.doi.org/ 10.14488/1676-1901.v16i1.2031 }}$

Revista Produção Online, Florianópolis, SC, v.16, n. 1, p. 126-150, jan./mar. 2016. 